\title{
On the Jost Solution and Its Properties of Quantum Difference Equations with Hyperbolic Eigenparameter
}

\author{
Yelda Aygar \\ Ankara University, Faculty of Science, Department of Mathematics, 06100, Ankara, Turkey
}

\begin{abstract}
This work is devoted to get Jost solution by the method of variation of parameters and properties of this Jost solution of a boundary value problem (BVP) depending on an hyperbolic eigenparameter. The results obtained are then used to find the Green function, resolvent and continuous spectrum of this BVP by using the methods of operator theory, methods of functional analysis and the general theory of difference equations. Moreover, using the Weyl compact perturbation theorem, we get that the operator $L$ generated by the $q$-difference expression has continuous spectrum filling the segment $[-2 \sqrt{q}, 2 \sqrt{q}]$.
\end{abstract}

\section{Introduction}

Quantum calculus which known as the calculus without limits is a connection between mathematics and physics. In recent years, it has led to rapid development of the theory of $q$-difference equations. The study of $q$ difference equations has become an important area of research. Because such equations have an interest role due to their applications in several mathematical areas such as number theory, orthogonal polynomials, mathematical control theory, basic hyper-geometric functions and other disciplines including mechanic, theory of relativity, biology, economics. Since it has huge applications in several disciplines, spectral analysis of $q$ -difference equations and operators have become a new research area for mathematicians. Spectral analysis of self-adjoint and nonselfadjoint $q$-difference equations has been investigated in $[1,2,4,7]$ both ordinary and matrix cases. Also BVPs consisting of $q$-difference equations and boundary condition depending on spectral parameter is studied in [3,5]. Differently studies which are mentioned above, in this work the spectral analysis of a $q$-difference equation with boundary condition is investigated using hyperbolic eigenparameter. As a result of this, analytical region of Jost solution has changed from upper half-plane to left half-plane. Some different aspects on the appearance of Jost solution, resolvent and Green function are stand out. This new approach will provide a wide perspective on applications of these problems in physics, mathematics, economics and engineering. In this study, we let $q>1$ and use the notation

$$
q^{\mathbb{N}_{0}}:=\left\{q^{n}: n \in \mathbb{N}_{0}\right\},
$$

where $\mathbb{N}_{0}$ denotes the set of nonnegative integers. $\ell_{2}\left(q^{N}\right)$ is a Hilbert space of complex-valued functions with the inner product

$$
\langle f, g\rangle_{q}:=\sum_{t \in q^{\mathbb{N}_{0}}} \mu(t) f(t) \overline{g(t)}, \quad f, g: q^{\mathbb{N}_{0}} \rightarrow \mathbb{C}
$$

and the norm

$$
\|f\|_{q}:=\left(\sum_{t \in q^{\mathbb{N}_{0}}} \mu(t)|f(t)|^{2}\right)^{\frac{1}{2}}, f: q^{\mathbb{N}_{0}} \rightarrow \mathbb{C}
$$

where $\mu(t)=(q-1) t$ for all $t \in q^{\mathbb{N}_{0}}$. Let us consider the nonselfadjoint BVP consisting of the second-order $q$ -difference equation

$q a(t) y(q t)+b(t) y(t)+a\left(\frac{t}{q}\right) y\left(\frac{t}{q}\right)=\lambda y(t), \quad t \in q^{\mathbb{N}}$

and the boundary conditions

$$
\begin{gathered}
\left(\gamma_{0}+\gamma_{1} \lambda\right) y(q)+\left(\beta_{0}+\beta_{1} \lambda\right) y(1)=0 \\
\gamma_{0} \beta_{1}-\gamma_{1} \beta_{0} \neq 0, \quad \gamma_{1} \neq \frac{\beta_{0}}{a(1)}
\end{gathered}
$$

where $\{a(t)\}_{t \in q^{\mathbb{N}_{0}}}$ and $\{b(t)\}_{t \in q^{\mathbb{N}}}$ are complex sequences, $\lambda$ is an eigenparameter, $a(t) \neq 0$ for all $t \in q^{N_{0}}$, and $\gamma_{i}, \beta_{i} \in \mathbb{C}, i=0,1$. In this work, we 
discuss the Jost solution and Jost function of of the BVP (1)-(2) and after getting its Green function and Resolvent, we investigate the continuous spectrum of this BVP under the assumption

$$
\sum_{t \in q^{\mathbb{N}}}(|1-a(t)|+|b(t)|)<\infty .
$$

\section{Main results}

Under the condition, we get the solution of BVP (1)-(2) as

$$
e(t, z)=\alpha(t) \frac{e^{\frac{\ln t}{\ln q}}}{\sqrt{\mu(t)}}\left(1+\sum_{r \in q^{\mathbb{N}}} A(t, r) e^{\frac{\ln r}{\ln z}}\right), t \in q^{\mathbb{N}_{0}}
$$

for $\lambda=2 \sqrt{q} \cosh z$ by using the similar method given in $\quad[5], \quad$ where $\quad z \in \overline{\mathbb{C}}_{-}:=\{z \in \mathbb{C}: z \leq 0\}$ $\mu(t)=(q-1) t$ for all $t \in q^{\mathbb{N}_{0}}$ and $\alpha(t), A(t, r)$ are expressed in terms of $\{a(t)\}$ and $\{b(t)\}$ as

$$
\begin{gathered}
\alpha(t)=\prod_{s \in[t, \infty) \cap q^{\mathbb{N}}}[a(s)]^{-1}, \\
A(t, q)=-\frac{1}{\sqrt{q}} \sum_{s \in[q t, \infty) \cap q^{\mathbb{N}}} b(s), \\
A\left(t, q^{2}\right)=\sum_{s \in[q t, \infty) \cap q^{\mathbb{N}}}\left\{1-a^{2}(s)+\frac{b(s)}{q} \sum_{p \in[q s, \infty) \cap q^{\mathbb{N}}} b(s)\right\}, \\
+\sum_{s \in[q t, \infty) \cap q^{\mathbb{N}}}\left\{\left(t, r q^{2}\right)=A(q t, r)\right.
\end{gathered}
$$

for $t \in q^{\mathbb{N}_{0}}$ and $r \in q^{\mathbb{N}}$. Moreover it is given in [4] that $A(t, r)$ satisfies

$$
|A(t, r)| \leq C \sum_{\left.s \in\left[t q^{\left[\frac{\ln r}{2 \ln q}\right]}\right], \infty\right) q^{\mathbb{N}}}(|1-a(s)|+|b(s)|),
$$

where $\left\lfloor\frac{\ln r}{2 \ln q}\right\rfloor$ is the integer part of $\frac{\ln r}{2 \ln q}$ and $C>0$ is a constant. Using (3) it is seen that $e(\cdot, z)$ is analytic with respect to $z$ in $\mathbb{C}_{-}:=\{z \in \mathbb{C}: z<0\}$ and continuous in $\overline{\mathbb{C}}_{-}$. Using (4) = and the boundary condition, we define the function $g$ by

$$
\begin{aligned}
g(z)= & \left(\gamma_{0}+2 \sqrt{q} \gamma_{1} \cosh z\right) e(q, z) \\
+ & \left(\beta_{0}+2 \sqrt{q} \beta_{1} \cosh z\right) e(1, z) .
\end{aligned}
$$

The function $g$ is analytic in $\mathbb{C}_{-}$, continuous in $\overline{\mathbb{C}}_{-}$, and $g(z)=g(z+2 \pi)$. Analogously to the Sturm-Liouville differential equation, the solution $e(\cdot, z)$ and the function $g$ are called the Jost solution and Jost function of (1)-(2), respectively [6]. Let $\phi(\lambda)=\{\phi(t, z)\} \quad t \in q^{N_{0}}$, be the solution of (1) satisfying the initial conditions

$$
\phi(1, \lambda)=-\left(\gamma_{0}+\gamma_{1} \lambda\right), \quad \phi(q, \lambda)=\left(\beta_{0}+\beta_{1} \lambda\right) .
$$

If we define

$$
\varphi(t, z)=\phi(2 \sqrt{q} \cosh z)=\{\phi(t, 2 \sqrt{q} \cosh z)\}_{t \in q^{\mathbb{N}_{0}}},
$$

then $\varphi$ is an entire function and $\varphi(z)=\varphi(z+2 \pi)$. Let us take the semi-strips $P_{0}-\left\{z \in \mathbb{C}_{-}:-\frac{\pi}{2} \leq z \leq \frac{3 \pi}{2}\right\}$

and $P=P_{0} \cup\left[-\frac{i \pi}{2}, \frac{3 i \pi}{2}\right]$. The Wronskian of two solutions $y=\{y(t, \lambda)\}_{t \in q^{\mathbb{N}}} \quad$ and $\quad v=\{(t, \lambda)\}_{t \in q^{\mathbb{N}}}$ of $\quad(1)$ is defined by

$W[y, v]=\mu(t) a(t)\left\{y(t, \lambda)_{V}(q t, \lambda)-y(q t, \lambda)_{V}(t, \lambda), \quad t \in q^{N_{0}}\right.$.

Hereafter, we find

$$
W[\varphi(t, z), e(t, z)]=-\mu(1) a(1) g(z) .
$$

Since $\varphi(\cdot, Z)$ and $e(\cdot, Z)$ are independent solutions for all $z \in P$ with $g(z) \neq 0$, we find the Green function of the BVP (1)-(2)

$$
G_{t, r}(z):=\left\{\begin{array}{lll}
-\frac{\varphi(r, z) e(t, z)}{q a(1) f(z)}, & r=t q^{-k}, & k \in \mathbb{N}_{0} \\
-\frac{e(r, z) \varphi(t, z)}{q a(1) f(z)}, & r=t q^{k}, & k \in \mathbb{N} .
\end{array}\right.
$$

It is clear that

$$
R_{\lambda} h(t):=\sum_{r \in q^{\mathbb{N}}} G(t, r) h(r), \quad h \in \ell_{2}\left(q^{\mathbb{N}}\right)
$$

is the resolvent of the BVP (1)-(2). Related to the equation (1), we will recall the $q$-difference operator by $L$ generated in $\ell_{2}\left(q^{\mathbb{N}}\right)$ by the $q$-difference expression

$(1 y)(t):=a\left(\frac{t}{q}\right) y\left(\frac{t}{q}\right)+b(t) y(t)+q a(t) y(q t), \quad t \in q^{N}$.

Note that (1) can be rewritten as $(l y)(t)=\lambda y(t)$. Let $L_{1}$ and $L_{2}$ denote the $q$-difference operators generated in $\ell_{2}\left(q^{N}\right)$ by

$$
\left(I_{1} y\right)(t):=y\left(\frac{t}{q}\right)+q y(q t), \quad t \in q^{N}
$$

and

$\left(I_{2} y\right)(t):=\left(a\left(\frac{t}{q}\right)-1\right) y\left(\frac{t}{q}\right)+b(t) y(t)+q(a(t)-1) y(q t), \quad t \in q^{N}$, respectively. It is clear that $L_{1}$ is a self-adjoint operator 
and $L=L_{1}+L_{2}$ [4]. It can be easily seen that $\frac{e^{\frac{\ln t}{\ln } z}}{\sqrt{\mu(t)}}$ and $\frac{e^{-\frac{\ln t}{\ln q}}}{\sqrt{\mu(t)}}$ are linearly independent solutions of $\left(l_{1} y\right)(t)=\lambda y(t) \quad$ for $\quad \lambda=2 \sqrt{q} \cosh z \quad$. The Wronskian of these two solutions is

$$
W\left[\frac{e^{\frac{\ln t}{\ln q}}}{\sqrt{\mu(t)}}, \frac{e^{-\frac{\ln t}{\ln q}}}{\sqrt{\mu(t)}}\right]--\frac{2}{\sqrt{q} \sinh z}, \quad z \in \mathbb{C}_{-} .
$$

For all $z \in \mathbb{C}_{-} \backslash\{i k \pi: k \in \mathbb{Z}\}$, we define the green function and resolvent of $L_{1}$ by

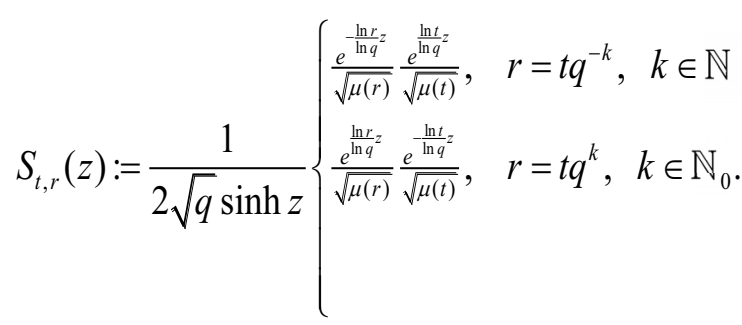

and

$$
R_{\lambda}\left(L_{1}\right) \psi(t):=\sum_{r \in q^{\mathbb{N}}} S(t, r) \psi(r), \quad \psi \in \ell_{2}\left(q^{\mathbb{N}}\right)
$$

respectively.

Lemma 1. For every $\delta>0$, there is a number such that $C_{\delta}$ such that

$$
\left\|R_{\lambda}\left(L_{1}\right)\right\|_{q}>\frac{C_{\delta}}{|\sinh z| \sqrt{1-e^{2 z}}}
$$

for all $z \in\left\{z \in P_{0}:|z|<\delta\right\}$.

Proof Let us define the function

$$
f(r, z):=\left\{\begin{array}{lll}
\frac{e^{-\frac{\ln r}{\ln q}}}{\sqrt{\mu(r)}}, & r-t q^{-k}, & k \in \mathbb{N} \\
0, & r-t q^{k}, & k \in \mathbb{N}_{0},
\end{array}\right.
$$

where $z=x+i y$. Thus, we obtain

$$
\| f\left(\cdot, z \|_{q}^{2}=\sum_{r \in\left(0, \frac{t}{q}\right] \cap q^{\mathbb{N}}}\left|e^{-\left.\frac{\ln r}{\ln q}\right|^{2}}=\sum_{r \in\left(0, \frac{t}{q}\right] \cap q^{\mathbb{N}}}\right| e^{-2 \frac{\ln r}{\ln q}} \mid<\infty .\right.
$$

Since $\|f(\cdot, z)\|_{q}^{2}<\infty, f(\cdot, z) \in \ell_{2}\left(q^{\mathbb{N}}\right)$. Using the (8) and (9), we get

$$
R_{\lambda}\left(L_{1}\right) f(\cdot, z)-\frac{e^{\frac{\ln t}{\ln q}}}{2 \sqrt{q} \sqrt{\mu(t)} \sinh z}\|f(\cdot, z)\|_{q}^{2} .
$$
that

Since $\left|e^{\frac{\ln t}{\ln q} z}\right|-\left|e^{\frac{\ln t}{\ln q}}\right|>\frac{1}{2} e^{\frac{\ln t}{\ln q} z}$, we obtain

$$
\begin{aligned}
&\left\|R_{\lambda}\left(L_{1}\right) f(\cdot, z)\right\|_{q}^{2}=\frac{\|f(\cdot, z)\|_{q}^{4}}{4 q|\sinh z|^{2}} \sum_{t \in q^{\mathbb{N}}}\left|e^{\frac{\ln r}{\ln q}}\right|^{2} \\
& \geq \frac{\|f(\cdot, z)\|_{q}^{4}}{16 q|\sinh z|^{2}} \sum_{t \in q^{\mathbb{N}}}\left(e^{\frac{\ln r}{\ln q}}\right)^{2} \\
&=\frac{\|f(\cdot, z)\|_{q}^{4}}{16 q|\sinh z|^{2}} \sum_{t \in q^{\mathbb{N}}} e^{2 \frac{\ln r}{\ln q} z} \\
& \geq \frac{\|f(\cdot, z)\|_{q}^{4}}{16 q|\sinh z|^{2}} \sum_{t \in(q, \infty) \cap q^{\mathbb{N}}}\left(e^{2 z}\right)^{\frac{\ln t}{\ln q}} \\
&=\frac{\|f(\cdot, z)\|_{q}^{4} e^{2 z}}{16 q|\sinh z|^{2}\left(1-e^{2 z}\right)} . \\
& \frac{\|f(\cdot z)\|_{q}^{2} e^{2 z}}{4 \sqrt{q}}, \text { the proof is complete. }
\end{aligned}
$$

Theorem 1. $\sigma\left(L_{1}\right)=\sigma_{\mathrm{c}}\left(L_{1}\right)=[-2 \sqrt{q}, 2 \sqrt{q}]$, where $\sigma\left(L_{1}\right)$ and $\sigma_{\mathrm{c}}\left(L_{1}\right)$ denote the spectrum and continuous spectrum of the operator $L_{1}$, respectively.

Proof. Because of the operator $L_{1}$ has no eigenvalues, the spectrum of it consists of only its continuous spectrum. If $\lambda_{0}=2 \sqrt{q} \cosh z_{0} \in \sigma_{\mathrm{c}}\left(L_{1}\right)$, then $\left\|R_{\lambda_{0}}\left(L_{1}\right)\right\| \rightarrow \infty$. Using (8) and (9), we have $z_{0} \rightarrow 0 \quad$. If $z_{0} \rightarrow 0$, then $\lambda_{0}=2 \sqrt{q} \cosh z_{0} \in[-2 \sqrt{q}, 2 \sqrt{q}]$. It follows from Lemma $\quad 1$ that $\left\|R_{\lambda}\left(L_{1}\right)\right\|_{q} \rightarrow \infty \quad$ for $\lambda=2 \sqrt{q} \cosh z \in[-2 \sqrt{q}, 2 \sqrt{q}]$. Now, if we show the range $\mathfrak{R}\left(L_{1}-\lambda I\right)$ of values of the operator $L_{1}-\lambda I$ is dense in $\ell_{2}\left(q^{N}\right)$, the proof is completed. The orthogonal complement of $\mathfrak{R}\left(L_{1}-\lambda I\right)$ coincides with the space of solutions of the equation $\left(l_{1}^{*} y\right)(t)=\lambda y$ such that $y \in \ell_{2}\left(q^{N}\right)$. Since the operator $L_{1}$ is a selfadjoint operator and has no eigenvalues, the orthogonal complement of the set $\mathfrak{R}\left(L_{1}-\lambda I\right)$ consists only of the zero element. It gives that the range $\mathfrak{R}\left(L_{1}-\lambda I\right)$ is dense in $\ell_{2}\left(q^{\mathbb{N}}\right)$.

Note that the different method of the proof of this theorem is given in [4].

Theorem 2. Under the condition (3), we have $\sigma_{\mathrm{c}}(L)=[-2 \sqrt{q}, 2 \sqrt{q}]$. 
Proof. Under the assumption (3), it is found that $L_{2}$ is a compact operator in $\ell_{2}\left(q^{N}\right)$ (see [8]). Then using Theorem 1 and the Weyl theorem [9] of a compact perturbation, it is seen that

$$
\sigma_{\mathrm{c}}(L)=\sigma\left(L_{1}\right)=\sigma_{\mathrm{c}}\left(L_{1}\right) \text {. }
$$

\section{Conclusion}

In this study, first we give the Jost solution of the BVP (1)-(2), then using the asymptotic behavior and analytical properties of the Jost solution, we obtain the Green fuction and the resolvent of BVP (1)-(2). At the end, we show that the continuous spectrum of the operatör is filling the segment $[-2 \sqrt{q}, 2 \sqrt{q}]$ under the condition (3). The properties of eigenvalues and spectral singularities of this BVP can be invastigated in the further research. For example, a condition can be given which guarante that this BVP has finite eigenvalues and spectral singularities with finite multiplicities.

\section{References}

1. M. Adıvar, M. Bohner, Spectral analysis of $q$ difference equations with spectral singularities, Math. Comp. Model. 43 (2006), 695703.

2. Y. Aygar, Investigation of spectral analysis of matrix quantum difference equations with spectral singularities, Hacettepe Journal of Mathematics and Statistics (2015), DOI:10.15672/HJMS.20164513107

3. Y. Aygar, Principal vectors of second-order quantum difference equations with boundary conditions dependent on spectral parameter, Advances in Difference Equations 2015:249 DOI:10.1186/s13662-015-0587-3.

4. Y. Aygar, M. Bohner, A Polynomial-Type Jost Solution and Spectral Properties of a Self-Adjoint Quantum-Difference Operator, Complex Analysis and Operator Theory, (2015) DOI:10.1007/s11785015-0463-x.

5. Y. Aygar, M. BohneR, On the spectrum of eigenparameter-dependent quantum difference equations, Appl. Math.\& Inf. Scie. 9 (2015), 17251729.

6. M.A. Naimark, Linear differential operators. Part II: Linear differential operators in Hilbert space, Frederick Ungar Publishing Co., New York, 1968.

7. Y. Aygar, M. Bohner, Spectral Anlaysis of a matrixvalued quantum difference operator, Dynm. Equ. Appl. (2015), In press.

8. L.A. Lusternik, V.J. Sobolev, Elements of functional analysis, Hindustan Publishing Corp., Delhi, 1974.

9. I.M. Glazman, Direct methods of qualitative spectral analysis of singular differential operators, Israel Program for Scientific Translations, Jerusalem, (1965) 